\section{Expression of lumican related to CD34 and VEGF in the articular disc of the human temporomandibular joint}

\author{
N. Kiga, I. Tojyo, T. Matsumoto, \\ Y. Hiraishi, Y. Shinohara, S. Fujita \\ Department of Oral and Maxillofacial \\ Surgery, Wakayama Medical University, \\ Wakayama City, Japan
}

\begin{abstract}
Lumican belongs to the small leucine-rich repeat proteoglycan (SLRP) gene family and has been reported to exist in the cornea, intervertebral disc and tendon. Lumican plays a significant role in the assembly and regulation of collagen fibres. The human temporomandibular joint (TMJ) disc is made up of fibrocartilage with an extracellular matrix (ECM) composed of collagen and proteoglycans. The existence and behaviour of lumican have not been studied in the human TMJ disc. Therefore, we used immunohistochemical methods to detect lumican, CD34 and vascular endothelial growth factor (VEGF) and histochemical staining with toluidine blue in 13 human TMJ specimens (10 surgically removed and 3 obtained from autopsy). In both normal and deformed discs we observed staining with toluidine blue. We found that the area of metachromasia inside the deformed disc was uneven and expression of lumican was strong in the areas negative for metachromasia. Staining of VEGF and CD34 inside the deformed disc was seen. We confirmed the expression of lumican in the human TMJ disc and showed that a large number of fibroblastlike cells existed in the area of strong lumican expression. These new findings about the behaviour of lumican suggest that it may play a key role in the generation of a new collagen network by fibroblast-like cells.
\end{abstract}

\section{Introduction}

In $1988, \operatorname{Scott}^{1}$ reported that proteokeratan sulfates (PKSs) were associated with collagen fibrils at specific binding sites. In 1991, Funderburgh et al. ${ }^{2}$ and Blochberger et $a .^{3}$ reported the existence of lumican in connective tissues. Lumican belongs to the family of SLRPs that plays a role in the ECM. The SLRP gene family has expanded in the past decade to encompass 17 genes. Recently, the gene family has been subdivided into 5 classes on sever- al parameters basis, including conservation and homology at the protein and genomic levels, the presence of characteristic N-terminal Cys-rich clusters with defined spacing and chromosomal organization. ${ }^{47}$ Lumican is classified as class 2 in the SLRP gene family. Lumican has been shown to exist in the connective tissue of many organs, including the cornea, intervertebral disc and tendon, and its behaviour has been studied in several clinical fields. ${ }^{8-12} \operatorname{Scott}^{13}$ have indicated that PKSs play a role in orienting and organizing the collagen fibrils, thus helping to establish and maintain tissue shape. Kafienah et al..$^{14}$ reported that lumican plays a role in limiting the growth of type II collagen fibrils during cartilage tissue engineering. Funderburgh et al. ${ }^{15}$ revealed that lumican is deeply involved in collagen fibre repair and adjustment, and Saika et al. ${ }^{16}$ showed that lumican expressed in injured epithelium may modulate cell behaviours such as adhesion or migration, thus contributing to corneal epithelial wound healing. In addition, Chakravarti et $a l^{17}$ revealed the early regulation of collagen structure by lumican during postnatal development of the cornea.

Extracellular matrix is composed mainly of collagen and proteoglycan in the articular disc of the TMJ. ${ }^{18,19}$ The articular disc lies between the condylar head and the glenoid fossa and helps to absorb force during opening and closing movements and occlusion. Chemical stress due to cytokines and physical stress due to malocclusion have been considered to be major causes of internal derangement (ID) and osteoarthritis (OA) in disorders of the TMJ. It is still unclear what kind of changes happens in the TMJ disc affected by ID and $\mathrm{OA}$ and no studies have examined the expression of lumican in the human TMJ disc with ID or $0 A$.

Based on the above, we hypothesised that lumican expression was likely to be increased in the deformed discs. Therefore, this study used histology and immunohistochemistry to compare human TMJ discs from normal and diseased individuals.

\section{Materials and Methods}

Thirteen human TMJ discs (10 diseased and 3 normal controls) were used in this study. The 10 diseased human TMJ specimens were surgically removed from 10 patients ( 8 female and 2 male) because of ID with severe pain and dysfunction. All the patients provided their informed consent, and this study was approved by the Ethics Committee of Wakayama Medical University.

The patients' age ranged from 20 to 72 years, with a mean age of 43.8 years (Table 1$)$.
Correspondence: Norifumi Kiga, Department of Oral and Maxillofacial Surgery, Wakayama Medical University, 811-1 Kimiidera, Wakayama City 641-8509, Japan - Tel/Fax: +81.73 .4410643$

E-mail: kibun@wakayama-med.ac.jp

Key words: TMJ disc, lumican, CD34, VEGF, immunohistochemistry, metachromasia.

Received for publication: 30 March 2010.

Accepted for publication: 3 June 2010.

This work is licensed under a Creative Commons Attribution 3.0 License (by-nc 3.0).

(C)Copyright N. Kiga et al., 2010

Licensee PAGEPress, Italy

European Journal of Histochemistry 2010; 54:e34 doi:10.4081/ejh.2010.e34

Magnetic resonance imaging revealed that all patients had anterior disc displacement without reduction. Most of the patients experienced severe pain and all patients experienced disturbances of function without reduction. The patients did not have a history of trauma to the TMJ, and none of them had ankylosis. The three normal TMJ disc specimens were obtained by autopsy from 3 separate female patients (average age 56.7 years) who did not have a clinical history of TMJ disturbances (fresh cadavers); they died of diseases not related to TMJ (a tongue cancer, a gastric cancer and a breast cancer). At the time of autopsy, it was confirmed that the patients did not suffer from anterior disc displacement or deformation. Microscopic examination of haematoxylin-eosin-stained sections did not reveal any pathological changes. All specimens were cut sagittally and immediately fixed overnight in phosphate buffered saline (PBS) solution containing 4\% paraformaldehyde, embedded in paraffin wax, and cut into $5 \mu \mathrm{m}$ thick sections.

\section{Immunohistochemical methods}

For immunohistochemical staining, the sections were dewaxed in xylene and rehydrated through an alcohol series. Endogenous peroxidase was blocked by immersing the sections in $0.3 \% \mathrm{H}_{2} \mathrm{O}_{2}$ in methanol for $20 \mathrm{~min}$ at room temperature. The specimens were blocked with $3 \%$ skimmed milk (in $0.01 \%$ Tween-PBS) for 60 min at room temperature and were then stained using a rabbit polyclonal primary antibody against lumican (Wakayama Medical University, Wakayama, Japan) at $4^{\circ} \mathrm{C}$ for $24 \mathrm{~h}$. The anti-lumican polyclonal antibody was used at a dilution of 1: 800. After washing with PBS, the specimens were treated with the secondary 
antibody using the EnVision ${ }^{\mathrm{TM}}+$ System-HRP Labelled Polymer, Anti-Rabbit (catalogue no. K4003, Dako Corporation, Tokyo, Japan) for 60 min at room temperature. Colour was developed using the Liquid DAB+ Substrate Chromogen System (catalogue no. K3468, Dako Corporation, Tokyo, Japan) followed by counterstaining with Mayer's haematoxylin (No.3000-2 Muto Pure Chemicals Corporation, Tokyo, Japan). For immunohistochemical staining of CD34 and vascular endothelial growth factor (VEGF), anti-human CD34 monoclonal antibody (Nichirei, Tokyo, Japan) and anti- human VEGF polyclonal antibody (Takara, Shiga, Japan) were used at a dilution of 1:5 and 1:3000, respectively. Negative controls were subjected to the same protocol but without the primary antibody.
Table 1. Characteristics of patients with temporomandibular disorders included in this study.

\begin{tabular}{ccclccc} 
Sample no. & Sex & Age & Diagnosis & \multicolumn{2}{c}{$\begin{array}{c}\text { Symptom } \\
\text { (pain) }\end{array}$} & $\begin{array}{c}\text { Displaement } \\
\text { of Tlys disc }\end{array}$ \\
\hline 1 & Female & 24 & ID and OA & + & + & + \\
2 & Female & 52 & ID and OA & + & + & + \\
\hline 3 & Female & 20 & ID and OA & + & + & + \\
4 & Male & 72 & ID and OA & - & + & + \\
\hline 5 & Female & 52 & ID and OA & - & + & + \\
6 & Male & 64 & ID and OA & - & + & + \\
\hline 7 & Female & 34 & ID and OA & - & + & + \\
8 & Female & 32 & ID and OA & + & + & + \\
\hline 9 & Female & 29 & ID and OA & + & + & + \\
10 & Female & 59 & ID and OA & + & + & + \\
\hline 11 & Female & 61 & Autopsy sample & - & - & + \\
\hline 12 & Female & 61 & Autopsy sample & - & - & + \\
\hline 13 & Female & 48 & Autopsy sample & - & - & + \\
\hline
\end{tabular}

A
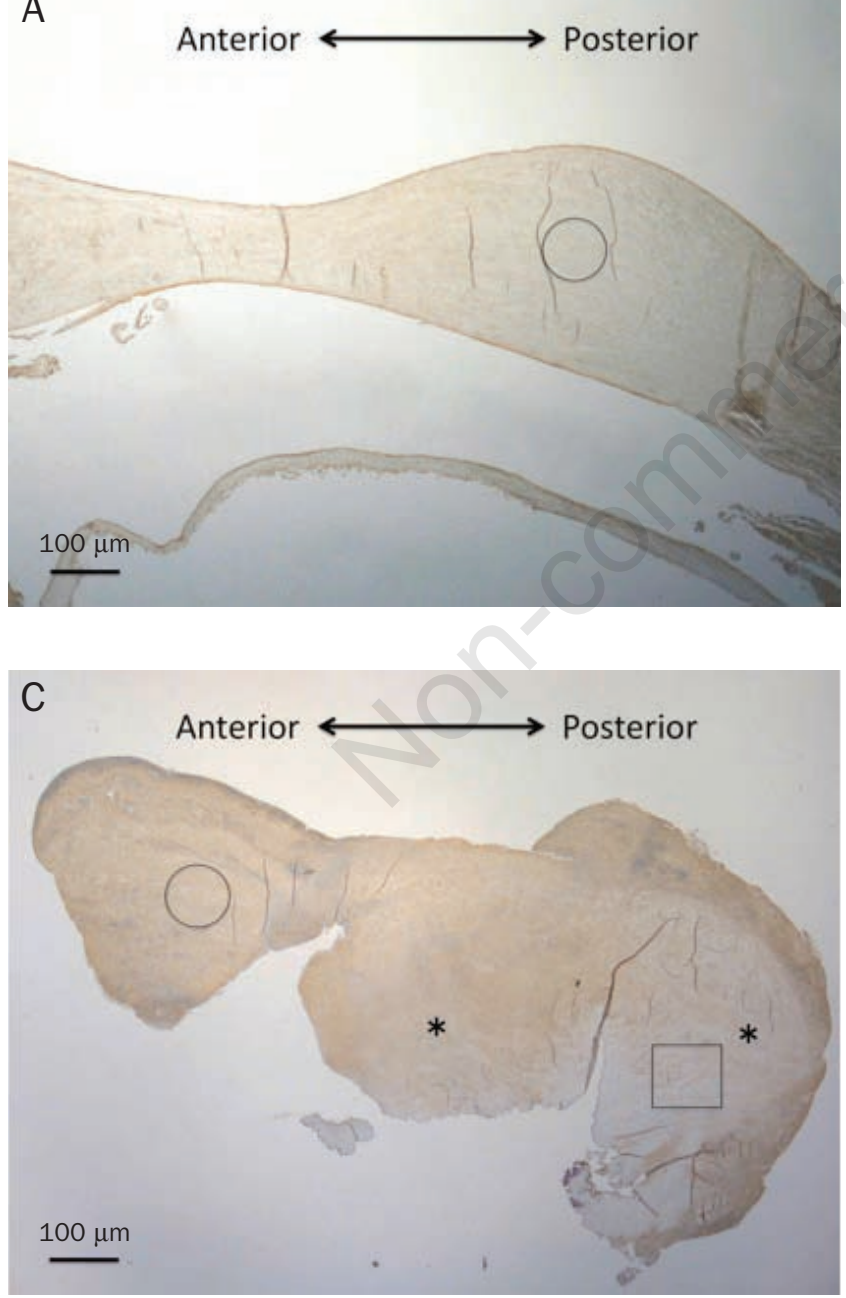

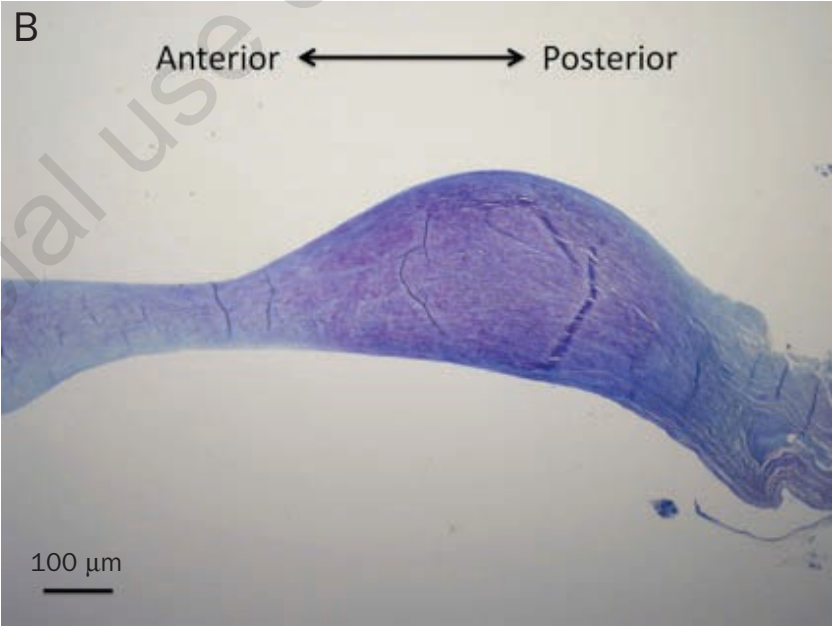

D

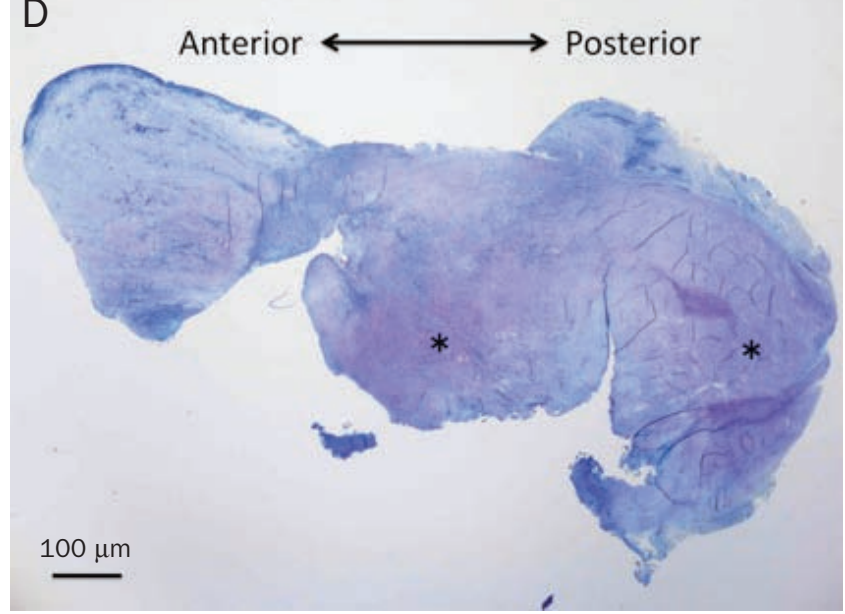

Figure 1. (A, B, C, D) Lumican expression and toluidine blue staining in the human TMJ disc (scale bar $=100 \mu \mathrm{m})$. (A) Lumican expression in the normal disc. (B) Metachromasia in the normal disc. (C) Strong lumican expression in the deformed disc (asterics: uneven or weak expression). (D) Metachromasia in the deformed disc (asterics: positive areas). All specimens are sagittal sections. 


\section{Histological methods}

Histological staining was performed using toluidine blue. Sections were dewaxed in xylene and rehydrated through an alcohol series. After washing with distilled water, sections were stained with $0.05 \%(\mathrm{w} / \mathrm{v})$ toluidine blue (pH 7.0) for $1 \mathrm{~min}$.

\section{Results}

Our findings in the human TMJ discs were as follows. To detect the localization of lumican in the whole human TMJ disc, immunohistochemical analysis was performed. The expression of lumican was detectable in both normal and deformed discs. In normal discs, positive staining of lumican was observed in the connective tissue around the disc and the disc surface, and weak staining for lumican observed inside the disc; whereas in deformed disc the strong expression also spread to the inside of the disc (Figure 1A, C). In both normal and deformed discs, metachromasia was detected by toluidine blue staining (Figure 1B, D).

In all discs we identified fibroblast-like cells, fibrochondrocytes, without a pericellular halo; and chondrocyte-like cells with rounded nuclei surrounded by a large halo of cytoplasm. In normal discs, the expression of lumican was weak surrounding all the cells (Figure 2A). In deformed discs, lumican was expressed in areas with many fibroblast-like cells (Figure 2B). On the other hand, the expression of lumican was weak surrounding fibrochondrocytes and chondrocyte-like cells (Figure 2C, D).

We analyzed the discs following staining with anti-human CD34 monoclonal antibody and anti-human VEGF polyclonal antibody. Positive staining of VEGF and CD34 within the deformed discs was observed (Figure 3A, C), which was not only found in normal discs (Figure 3B, D).

\section{Discussion}

Several recent studies have cast light on the localisation and function of lumican. ${ }^{8-12}$ Fibrilassociated molecules such as lumican have been implicated in regulating the progression of fibril
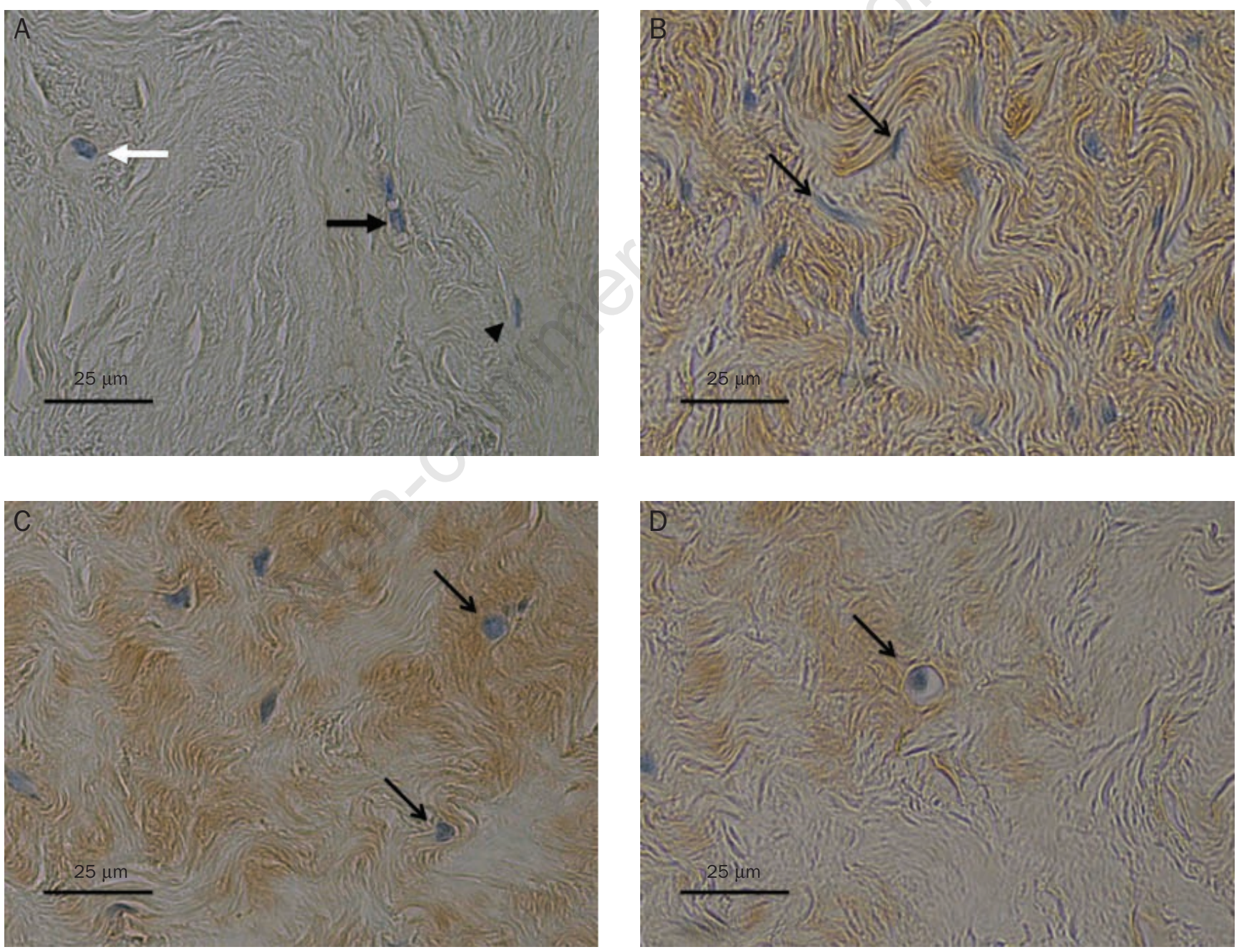

Figure 2. (A, B, C, D) Immunohistochemical staining of lumican in the TMJ discs (scale bar $=25 \mu \mathrm{m})$. (A) The fibroblast-like cells (arrowhead) and the fibrochondrocyte (black arrow), the chondrocyte-like cell (white arrow) in the normal disc (enlargement of circle in Figure 1A). (B) Fibroblast-like cells (arrows) (enlargement of circle in Figure 1C); (C) Fibrochondrocytes (arrows) (enlargement of circle in Figure 1C); (D) Chondrocyte-like cells (arrow) in the deformed disc (enlargement of rectangle in Figure 1C). All specimens are sagital sections. 
assembly through a series of steps leading to structurally and mechanically mature fibrils..$^{20}$ Electron microscopic investigations of lumicannull mice reveal deregulated growth of collagen fibrils with a significant proportion of abnormally thick fibrils in skin and cornea. ${ }^{10}$ Changing patterns of abundance of lumican observed with age suggest that the disc may have different requirements for the composition of its extracellular matrix at different stages of life. ${ }^{9}$ Although lumican has been studied in the cornea, tendon and intervertebral disc, very little has been reported on lumican in the TMJ disc.

In this study, we focused on the following points; comparison of the positive reactions of toluidine blue, CD34 and VEGF in the deformed discs, confirmation of lumican expression within the TMJ disc and its rela- tionship to disc cells.

Firstly, the most interesting finding was identified by toluidine blue staining. Metachromasia could be seen in most areas of both normal and deformed discs. However, areas showing strong metachromasia coincided with weak expression of lumican in all the discs. We found that the positive area of metachromasia in the inside of the deformed disc was uneven and expression of lumican was strong in the areas negative for metachromasia.

Toluidine blue binds to hyaluronic acid and glycosaminoglycans (GAGs). The large proteoglycans of the TMJ disc are aggrecan, ${ }^{21-23}$ which consist of a core protein covalently linked to more than 100 chondroitin sulphate side chains. ${ }^{24}$
In corneal lumican, it has been reported that three of five binding sites are acceptors for keratan sulphate. ${ }^{2,25,26}$ Thus, we inferred that GAGs-rich ECM decreases due to the deformation of TMJ discs, while GAGs-poor lumican increases with the change of ECM, so that differences in the areas positive for lumican and metachromasia may appear.

Secondly, we identified positive expression of CD34 and VEGF in the deformed discs and found that lumican positive areas could be observed not only on the surface but also spread to the inside of the disc. VEGF belongs to the platelet derived growth factor family. ${ }^{27}$ Angiogenesis is accompanied by the expression of angiogenic mitogen such as VEGF, which involves the formation of new blood vessels from pre-existing vessels to form a capil-
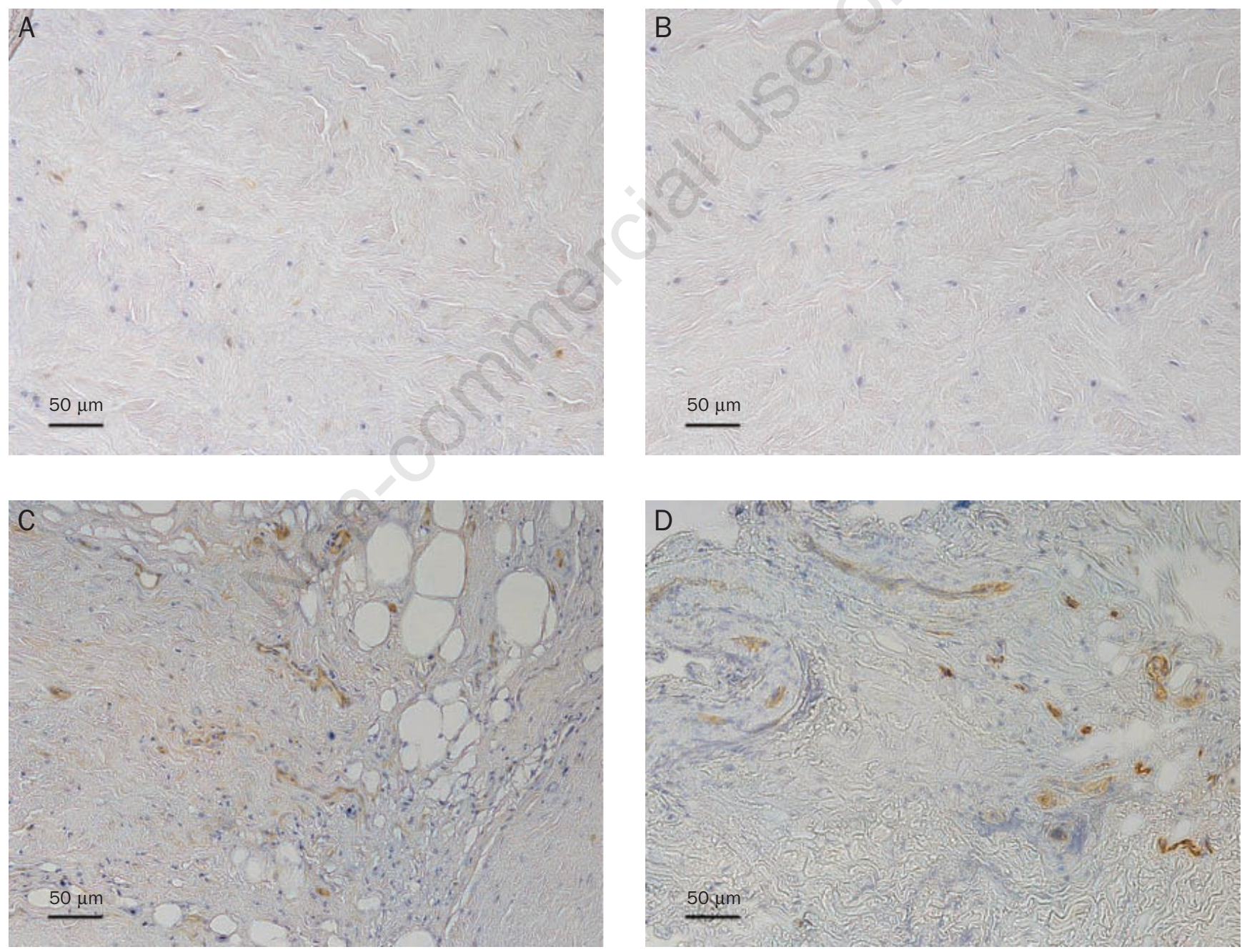

Figure 3. (A, B, C, D) Immunohistochemical staining of CD34 and VEGF in the TMJ discs (scale bar = 50 $\mu \mathrm{m})$. (A) Weak CD34 is observed sporadically within the normal disc. (B) Strong CD34 expression is observed in the wall of newly formed capillaries while weak expression is seen in the extracellular matrix. (C) VEGF expression was not found in the normal disc. (D) Strong VEGF expression is seen in the wall of newly formed capillaries. All specimens are sagital sections. 
lary networks. ${ }^{28,29} \mathrm{CD} 34$ is another transmembrane glycoprotein present on endothelial cells, leukemic cells, and some progenitor cells, which is produced by endothelial cells and associated with angiogenesis. ${ }^{30,31}$ Yoshida et $a l .^{31}$ reported that the presence of CD34 correlates with the process of angiogenesis induced by internal derangement of the TMJ and that new growth of blood capillaries is distributed in the internal derangement of human TMJ discs. ${ }^{31,32}$

Leonardi et al. reported that in normal discs, fibroblast-like cells, fibrochondrocytes and chondrocyte-like cells are either unstained or only weakly immunostained by VEGF antibody, while in disc specimens from internal derangement of the TMJ with significant tissue degeneration/regeneration, VEGF is consistently expressed..$^{33}$ Thus, we suggest that the spreading expression of lumican to the inside of the disc might be affected by inflammatory cytokines from blood capillaries.

The human TMJ disc has been said to contain fibroblast-like cells, fibrochondrocytes and chondrocyte-like cells. ${ }^{34}$ We confirmed that the large number of fibroblast-like cells co-localise with lumican expression in our specimens. In contrast, little lumican expression was observed in the vicinity of fibrochondrocytes and chondrocyte-like cells. In the current study lumican up-regulates before epithelial-mesenchymal transition (EMT) of the lens epithelial cells, which is characterized by a fibroblastlike morphology and by expression of $\alpha$ smooth muscle actin ( $\alpha \mathrm{SMA})$ and collagen type I. ${ }^{35,36}$ It has been suggested that the up-regulation of $\alpha$ SMA immunolabelling is associated with histopathological findings and in particular with an attempt for disc healing and plays an important role in the favourable evolution of a pathological phenomenon, such as in the case of deformed disc. ${ }^{37}$ Vij et al. revealed that Lumcan (-/-) mouse embryonic fibroblasts (MEF), compared to their wild type counterparts, display increased rates of proliferation and decreased apoptosis, and lumican appears to regulate these cellular functions by modulating specific cell growth and apoptosis mediators. ${ }^{38}$ For the reasons mentioned above, we suggested that expression of lumican may relate to an increase and a decrease of fibroblast-like cell in the TMJ disc and fibrosis of the deformed disc.

In summary, lumican expression may play a key role in the generation of a new collagen network by fibroblast-like cells. On the basis of these results, further research should be conducted to understand the involvement of mechanical stress acting on disc cells and proteoglycans in the TMJ disc and would be necessary to investigate a role for lumican in the regulation of collagen assembly using lumican-null mice. . $^{36,39}$

\section{References}

1. Scott JE. Proteoglycan-fibrillar collagen interactions. Biochem J 1988;252:313-23.

2. Funderburgh JL, Funderburgh ML, Mann MM, Conrad GW. Arterial lumican. Properties of a corneal-type keratan sulfate proteoglycan from bovine aorta. J Biol Chem 1991;266:24773-7.

3. Blochberger TC, Vergnes J, Hempel J, Hassell JR. cDNA to chick lumican (corneal keratan sulfate proteoglycan) reveals homology to the small interstitial proteoglycan gene family and expression in muscle and intestine. $\mathrm{J}$ Biol Chem 1992;267:347-52.

4. Schaefer L, Iozzo RV. Biological functions of the small leucine-rich proteoglycans: from genetics to signal transduction. J Biol Chem 2008;283:21305-9.

5. Nikitovic D, Katonis P, Tsatsakis A, Karamanos NK, Tzanakakis GN. Lumican, a small leucine-rich proteoglycan. IUBMB Life 2008;12:818-23.

6. Henry SP, Takanosu M, Boyd TC, Mayne PM, Eberspaecher H, Zhou W, et al. Expression pattern and gene characterization of asporin. A newly discovered member of the leucine-rich repeat protein family. J Biol Chem 2001;276:12212-21.

7. Iozzo RV. The biology of the small leucinerich proteoglycans. Functional network of interactive proteins. J Biol Chem 1999; 274:18843-6.

8. Funderburgh JL, Funderburgh ML, Brown SJ, Vergnes JP, Hassell JR, Mann MM et al. Sequence and structural implications of a bovine corneal keratan sulfate proteoglycan core protein. Protein 37B represents bovine lumican and proteins $37 \mathrm{~A}$ and 25 are unique. J Biol Chem 1993;268:11874-80.

9. Sztrolovics R, Alini M, Mort JS, Roughley PJ. Age-related changes in fibromodulin and lumican in human intervertebral discs. Spine 1999;24:1765-71.

10. Svensson L, Aszódi A, Reinholt FP, Fässler R, Heinegård D, Oldberg A. Fibromodulinnull mice have abnormal collage fibrils, tissue organization, and altered lumican deposition in tendon. J Biol Chem 1999; 274:9636-47.

11. Gill MR, Oldberg A, Reinholt FP. Fibromodulin-null murine knee joints display increased incidences of osteoarthritis and alterations in tissue biochemistry. Osteoarthritis Cartilage 2002;10:751-7.

12. Raouf A, Ganss B, McMahon C, Vary C, Roughley PJ, Seth A. Lumican is a major proteoglycan component of the bone matrix. Matrix Biol 2002;21:361-7.

13. Scott JE. Supramolecular organization of extracellular matrix glycosaminoglycans, in vitro and in the tissues. FASEB $\mathrm{J}$ 1992;6:2639-45.

14. Kafienah W, Cheung FL, Sims T, Martin I, Miot S, von Ruhland C, et al. Lumican inhibits collagen deposition in tissue engineered cartilage. Matrix Biol 2008;27:52634.

15. Funderburgh JL, Mitschler RR, Funderburgh ML, Roth MR, Chapes SK, Conrad GW. Macrophage receptors for lumican. A corneal keratan sulfate proteoglycan. Invest Ophthalmol Vis Sci 1997;38:115967.

16. Saika S, Shiraishi A, Liu CY, Funderburgh JL, Kao CW, Converse RL, et al. Role of lumican in the corneal epithelium during wound healing. J Biol Chem 2000;275: 2607-12.

17. Chakravarti S, Zhang G, Chervoneva I, Roberts L, Birk DE. Collagen fibril assembly during postnatal development and dysfunctional regulation in the lumican-deficient murine cornea. Dev Dyn 2006;235: 2493-506.

18. Piacentini C, Marchetti C, Bernasconi G, Menghini P, Baciliero U, Brusotti C. Collagen fiber arrangement in temporomandibular joint (TMJ) disks from human subjects with functional diseases. Scanning electron microscopy investigations. Scanning Microsc 1994;8:207-13.

19. Benjamin M, Ralphs JR. Biology of fibrocartilage cells. Int Rev Cytol 2004;233:1-45.

20. Ezura Y, Chakravarti S, Oldberg A, Chervoneva I, Birk DE. Differential expression of lumican and fibromodulin regulate collagen fibrillogenesis in developing mouse tendons. J Cell Biol 2000;151:779-88.

21. Nakano T, Imai S, Koga T, Dodd CM, Scott PG. Monoclonal antibodies to the large chondroitin sulphate proteoglycan from bovine temporomandibular joint disc. Matrix 1993;13:243-54.

22. Gu Z, Feng J, Shibata T, Hu J, Zhang Z. Type II collagen and aggrecan mRNA expression by in situ hybridization in rabbit temporomandibular joint posterior attachment following disc displacement. Arch Oral Biol 2003;48:55-62.

23. Allen KD, Athanasiou KA. Effect of passage and topography on gene expression of temporomandibular joint disc cells. Tissue Eng 2007;13:101-10.

24. Dunlevy JR, Rada JA. Interaction of lumican with aggrecan in the aging human sclera. Invest Ophthalmol Vis Sci 2004;45: 3849-56.

25. Dunlevy JR, Neame PJ, Vergnes J, Hassell JR. Identification of the N-linked oligosaccharide sites in chick corneal lumican and keratocan that receive keratan sulfate. J Biol Chem 1998;273:9615-21.

26. Melching LI, Roughley PJ. Modulation of 
keratan sulfate synthesis on lumican by the action of cytokines on human articular chondrocytes. Matrix Biol 1999;18:381-90.

27. Wauke K, Nagashima M, Ishiwata T, Asano G, Yoshino S. Expression and localization of vascular endothelial growth factor-C in rheumatoid arthritis synovial tissue. $\mathrm{J}$ Rheumatol 2002;29:34-8.

28. Nagashima M, Yoshino S, Ishiwata T, Asano G. Role of vascular endothelial growth factor in angiogenesis of rheumatoid arthritis. J Rheumatol 1995;22:162430 .

29. Fang W, Friis TE, Long X, Xiao Y. Expression of chondromodulin-1 in the temporomandibular joint condylar cartilage and disc. J Oral Pathol Med 2010;39: 356-60.

30. Delia BD, Lampugnani MG, Resnati M, Dejana E, Aiello A, Fontanella E, et al. CD34 expression is regulated reciprocally with adhesion molecules in vascular endothelial cells in vitro. Blood 1993;81:1001-8.
31. Yoshida H, Fujita S, Nishida M, Iizuka T. Angiogenesis in the human temporomandibular joint studied by immunohistochemistry for CD34 antigen. J Oral Pathol Med 1999;28:289-92.

32. Yoshida H, Fujita S, Nishida M, lizuka T. Localization of lymph capillaries and blood capillaries in human temporomandibular joint discs. J Oral Rehabil 1999;26:600-7.

33. Leonardi R, Lo Muzio L, Bernasconi G, Caltabiano C, Piacentini C, Caltabiano M. Expression of vascular endothelial growth factor in human dysfunctional temporomandibular joint discs. Arch Oral Biol 2003;48:185-92.

34. Leonardi R, Loreto C, Barbato E, Polimeni A, Caltabiano R, Lo Muzio L. A histochemical survey of the human temporomandibular joint disc of patients with internal derangement without reduction. J Craniofac Surg 2007;18:1429-33.

35. Saika S, Kono-Saika S, Ohnishi Y, Sato M, Muragaki Y, Ooshima A, et al. Smad3 signaling is required for epithelial-mes- enchymal transition of lens epithelium after injury. Am J Pathol 2004;164:651-63.

36. Saika S, Miyamoto T, Tanaka S, Tanaka T, Ishida I, Ohnishi Y, et al. Response of lens epithelial cells to injury: Role of lumican in epithelial- mesenchymal transition. Invest Ophthalmol Vis Sci 2003;44:2094-102.

37. Leonardi R, Villari L, Piacentini C, Bernasconi G, Travali S, Caltabiano C. Immunolocalization of vimentin and $\alpha$ smooth muscle actin in dysfunctional human temporomandibular joint disc samples. J Oral Rehabil 2002;29:282-6.

38. Vij N, Roberts L, Joyce S, Chakravarti S. Lumican suppresses cell proliferation and aids Fas-Fas ligand mediated apoptosis: implications in the cornea. Exp Eye Res 2004;78:957-71.

39. Chakravarti S, Magnuson T, Lass JH, Jepsen KJ, LaMantia C, Carroll H. Lumican regulates collagen fibril assembly: skin fragility and corneal opacity in the absence of lumican. J Cell Biol 1998;141:1277-86. 